\title{
Advances in Instrumentation and Monitoring in Geotechnical Engineering
}

\author{
D. N. Singh, ${ }^{1}$ Nagaratnam Sivakugan, ${ }^{2}$ and Sai K. Vanapalli ${ }^{3}$ \\ ${ }^{1}$ Department of Civil Engineering, Indian Institute of Technology Bombay, Mumbai 400076, India \\ ${ }^{2}$ Civil and Environmental Engineering, School of Engineering and Physical Sciences, James Cook University, \\ Townsville, QLD 4811, Australia \\ ${ }^{3}$ Civil Engineering Department, University of Ottawa, A112, 161 Louis Pasteur, Ottawa, ON, Canada K1N 6N5
}

Correspondence should be addressed to D. N. Singh, dns@civil.iitb.ac.in

Received 11 November 2011; Accepted 11 November 2011

Copyright (C) 2011 D. N. Singh et al. This is an open access article distributed under the Creative Commons Attribution License, which permits unrestricted use, distribution, and reproduction in any medium, provided the original work is properly cited.

Geotechnical instrumentation to monitor the performances of earth and earth-supported structures is increasingly becoming popular. Verification of long-term performances, validation of new theories, construction control, warning against any impending failures, quality assurance, and legal protection are some of the many reasons for geotechnical instrumentation. They are not only used in field situations, but in laboratories too. With the recent advances in materials and technology, and the need for more stringent performance control, there had been significant developments in the recent past in instrumentation and monitoring techniques.

We are thankful to Hindawi Publishing Corporation for inviting us to act as Guest Editors of this special issue. The main focus of this special issue is to document the recent advances in the instrumentation and monitoring techniques in geotechnical engineering. Authors were invited to disseminate their research findings and recent advances in the instrumentation and monitoring techniques in the field of geotechnical engineering. Apart from this, authors of about twenty papers, which were submitted to the 12th Conference of the International Association for Computer Methods and Advances in Geomechanics (IACMAG) held in Goa, India, from 1 to 6 October 2008, under the theme of "Geomechanics in the Emerging Social and Technological Age," were invited to upgrade/modify their manuscripts as per the requirements of the journal.

The paper titled "Real-time monitoring system and advanced characterization technique for civil infrastructure health monitoring" presents two successful field applications of the shape-acceleration array (SAA) system, at an active bridge realignment site in The Netherlands.

The paper titled "Nonparametric monitoring for geotechnical structures subject to long-term environmental change" presents a nonparametric, data-driven methodology of monitoring for geotechnical structures subject to long-term environmental change. For validating this methodology, data from a full-scale retaining wall, which has monitored for three years, has been used.

The paper titled "Field assessment and specification review for roller-integrated compaction" presents an overview of two technologies: compaction meter value (CMV) and machine drive power (MDP), an overview of factors influencing statistical correlations, modeling for visualization and characterization of spatial nonuniformity, and a brief review of the specifications being used by the professionals.

The paper titled "Experimental and numerical study of atrest lateral earth pressure of overconsolidated sand" presents an interesting experimental and numerical investigation of atrest lateral earth pressure resulted due to sandy soil adjacent to retaining walls.

The paper titled "Seeing through the ground: the potential of gravity gradient as a complementary technology" describes a multisensor device to locate buried services.

The paper titled "Stability evaluation of volcanic slope subjected to rainfall and freeze-thaw action based on field monitoring" aims at clarifying the aspects related to the stability of in situ volcanic slopes subjected to rainfall and freeze-thaw action. 
The paper titled "A methodology for determination of resilient modulus of asphaltic concrete" deals with development of a novel methodology that has been found to be quite useful for determining the resilient modulus of the material quite precisely.

The paper titled "A controllable approach to rectify inclined buildings" describes a safe construction and instrumentation technique to rectify the inclined buildings.

$$
\begin{array}{r}
\text { D. N. Singh } \\
\text { Nagaratnam Sivakugan } \\
\text { Sai K. Vanapalli }
\end{array}
$$

\section{Acknowledgments}

We are much thankful to the reviewers for extending their help, support, and guidance in bringing out this issue. We are thankful to the authors for extending their fullest cooperation in preparing the manuscripts as per the guidelines of the journal, taking into account the additional review comments. We acknowledge the IACMAG for approving publication of the following papers in this edition of the journal. 

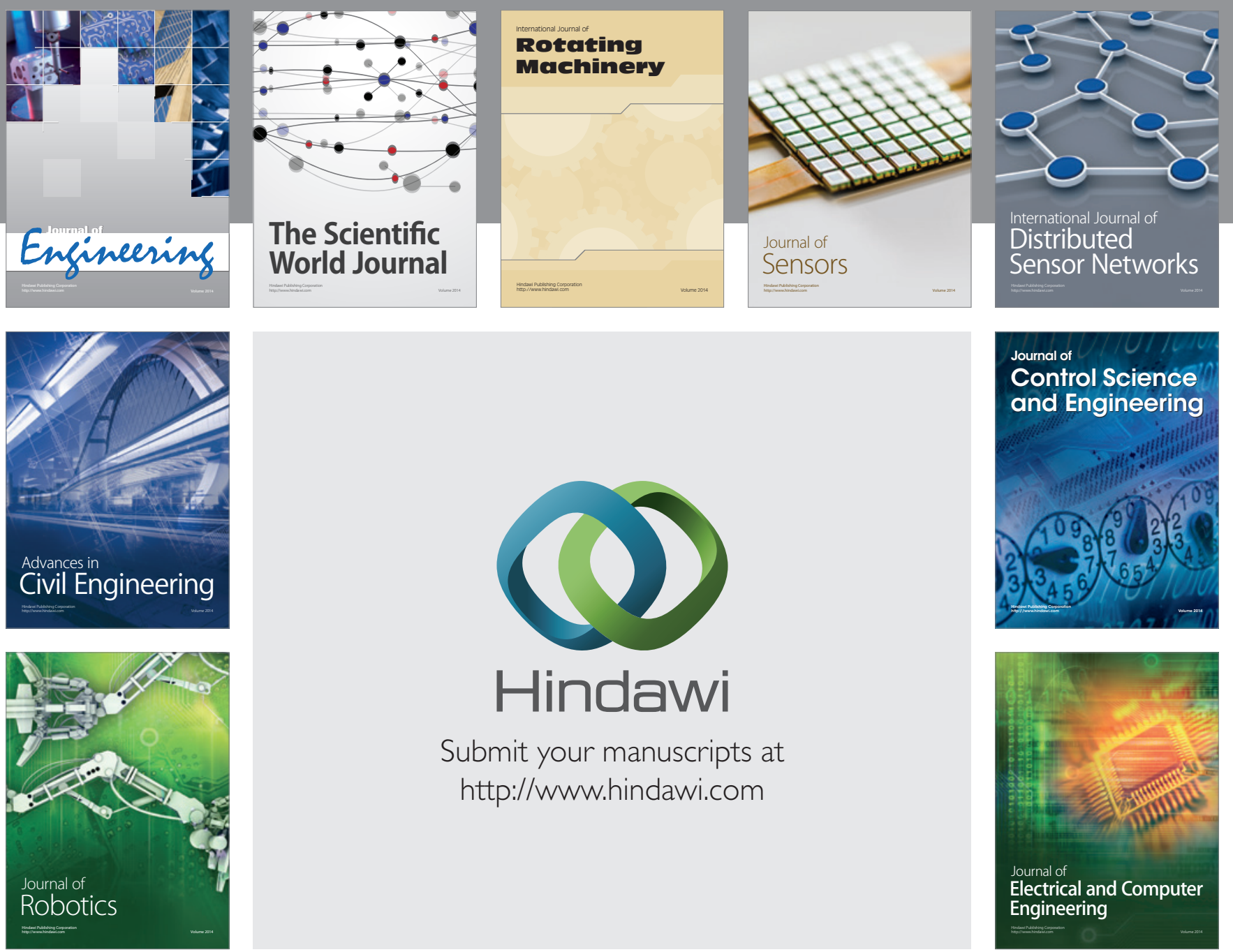

Submit your manuscripts at

http://www.hindawi.com
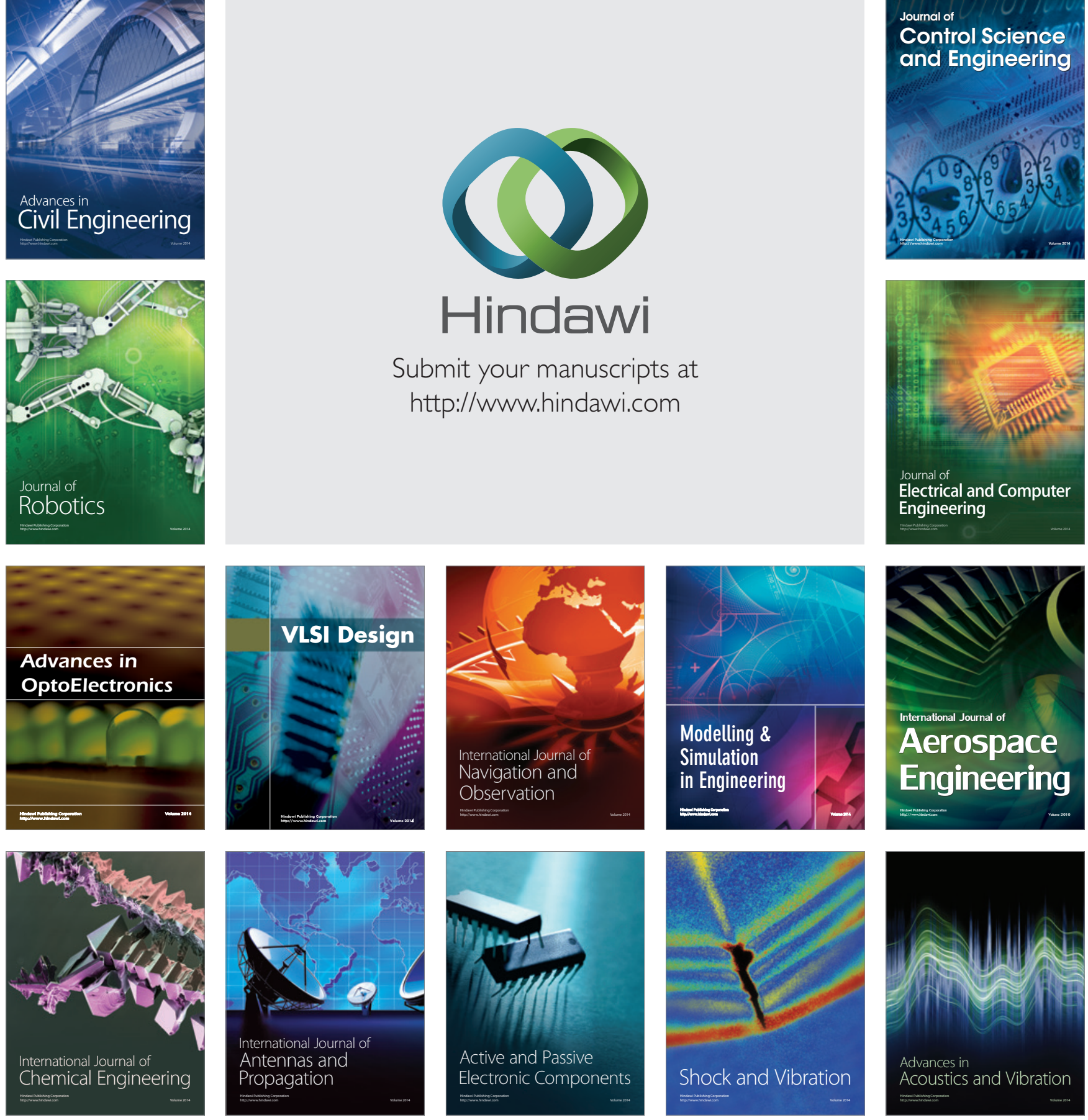